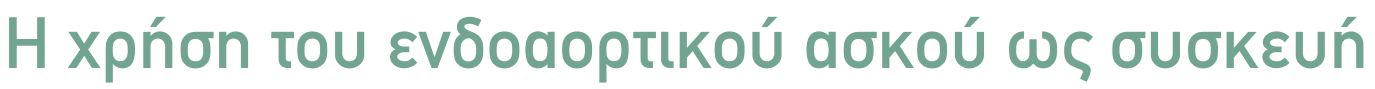

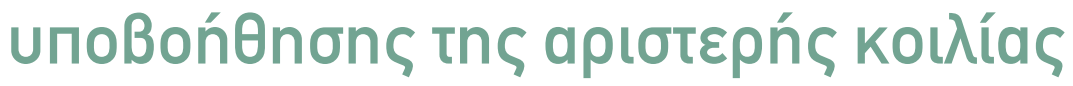

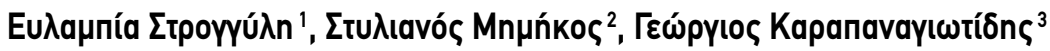

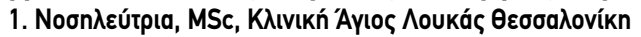

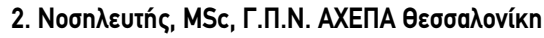

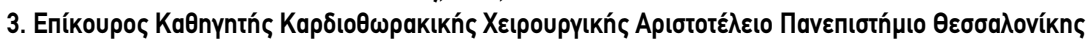

\section{ПEPIAHUH}

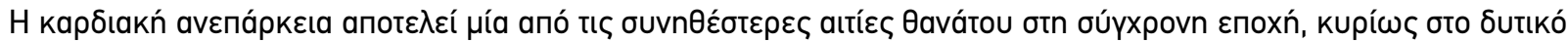

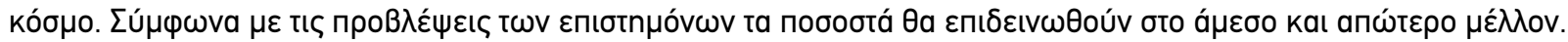

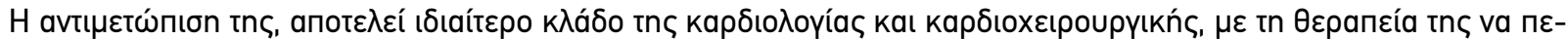

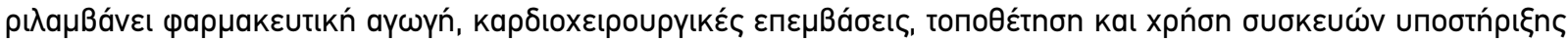

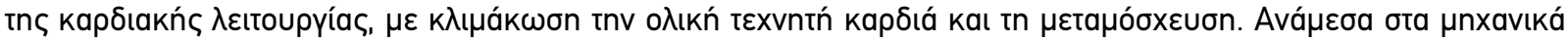

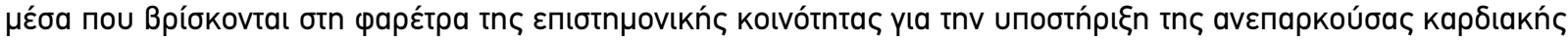

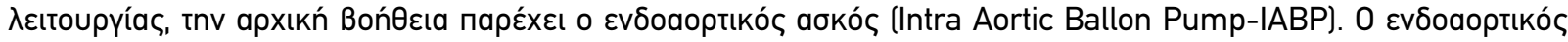

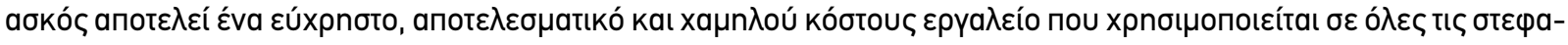

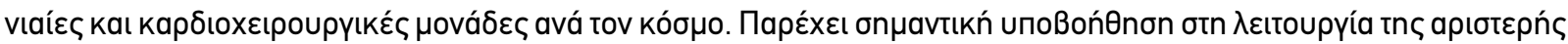

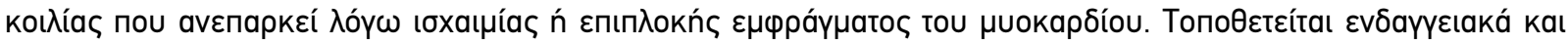

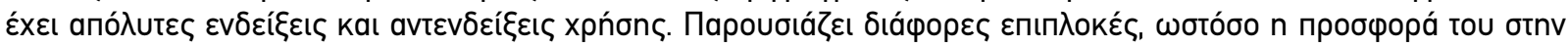

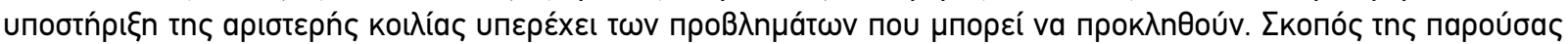

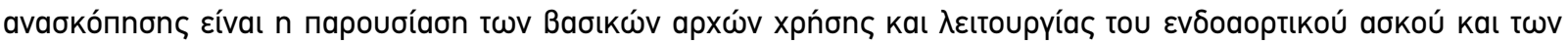

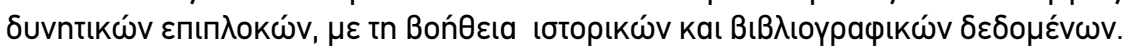

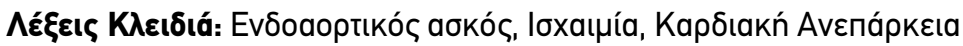

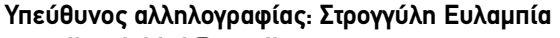
e-mail: evlabia1@gmail.com

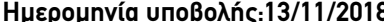

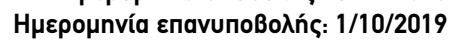

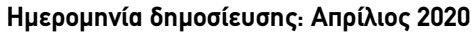

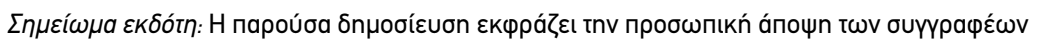

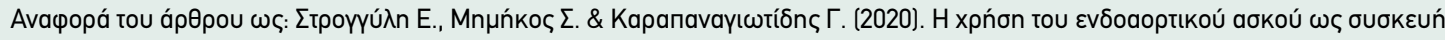

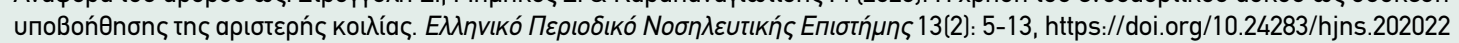




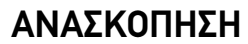

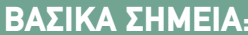

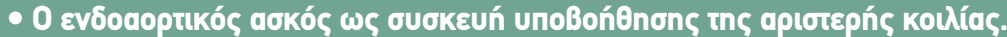

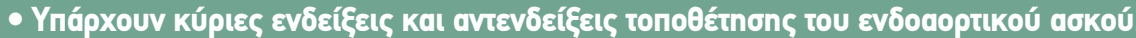

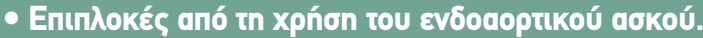

\section{EIIAГRГH}

$\mathrm{H}$

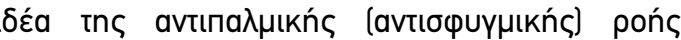

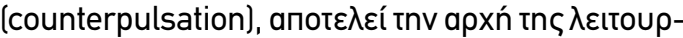

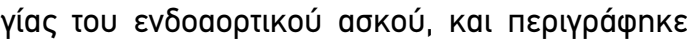

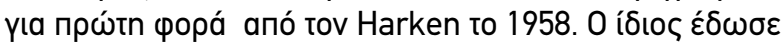

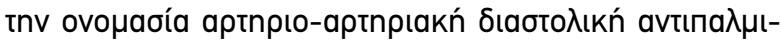
kń poń (Arterio-arterial diastolic counterpulsation). 0

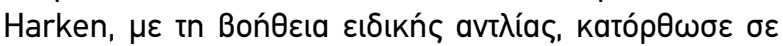

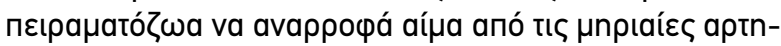

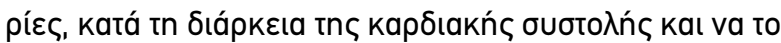

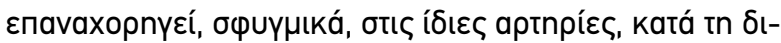

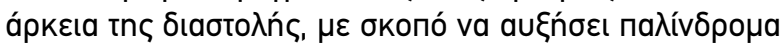

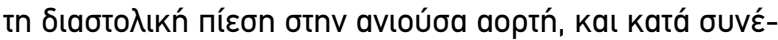

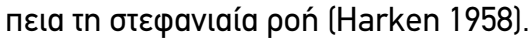

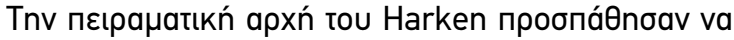

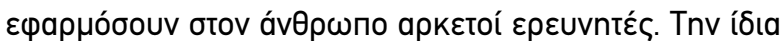

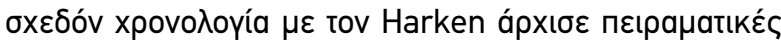

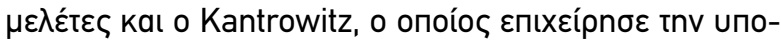

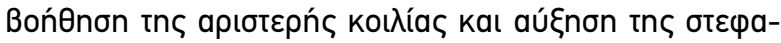

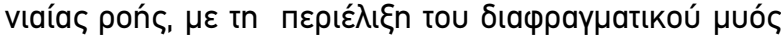

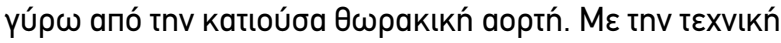

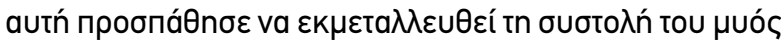

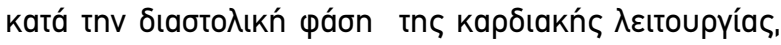

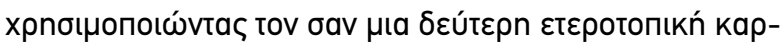
ठı́́ (Kantrowitz \& Mckinnon 1958).

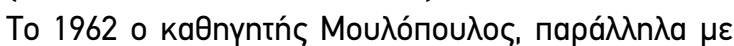

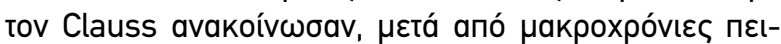

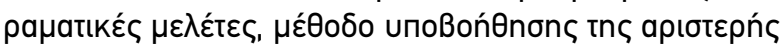

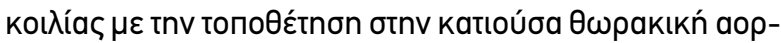

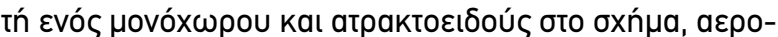

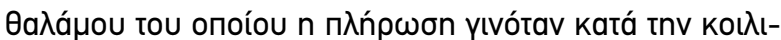

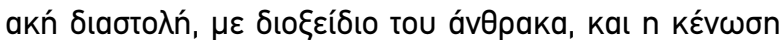

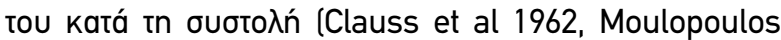

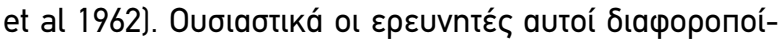

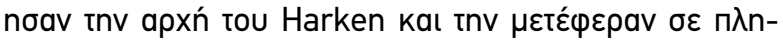

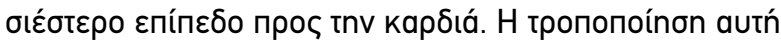

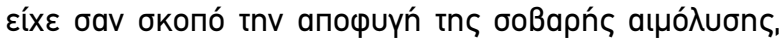

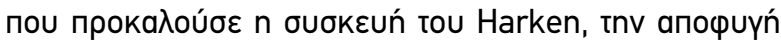

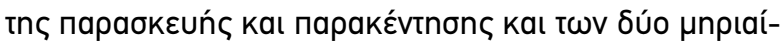

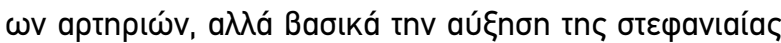

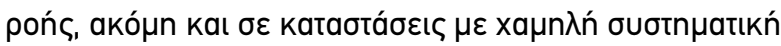

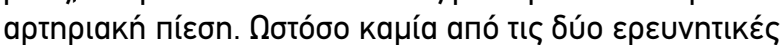

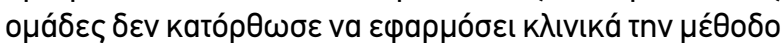
autń.

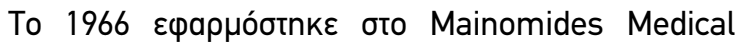

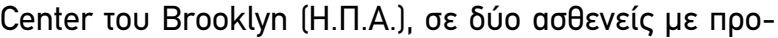

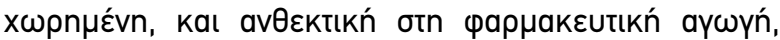

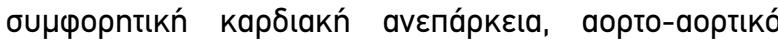

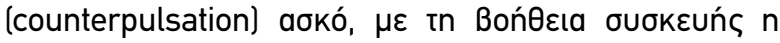

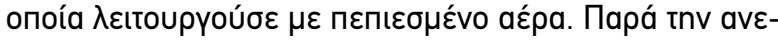

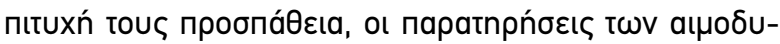

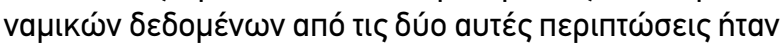

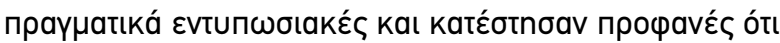

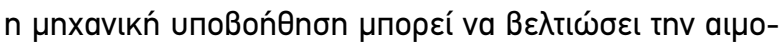

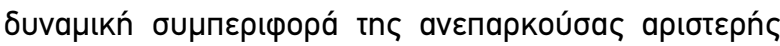
koı入ías (Kantrowitz et al 1966).

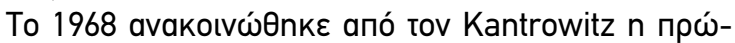

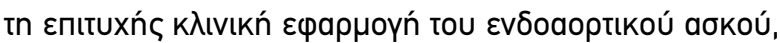

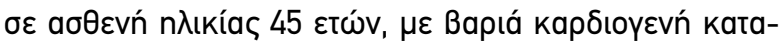

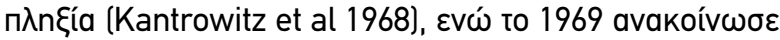

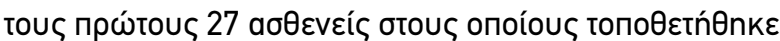

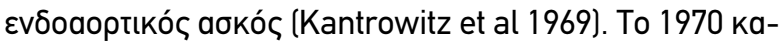

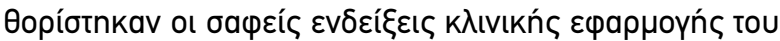

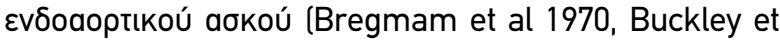

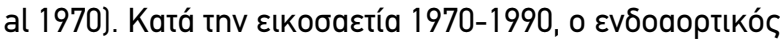

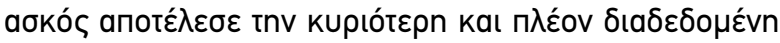

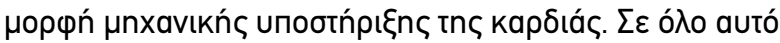

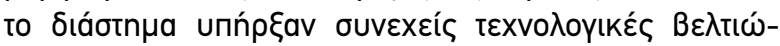

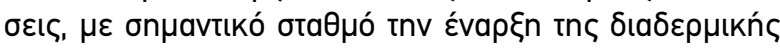

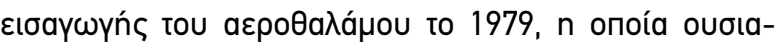

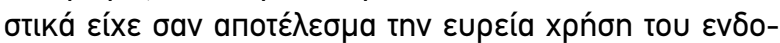

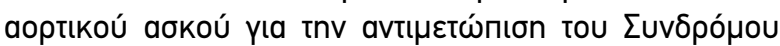

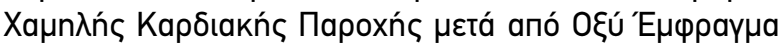

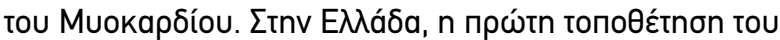

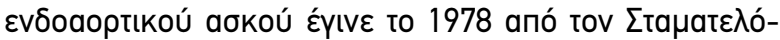

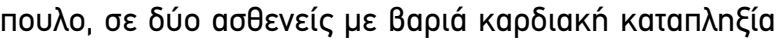

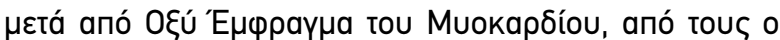

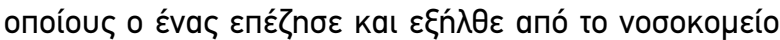
(Stamatelopoulos et al 1978).

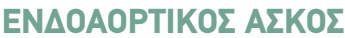

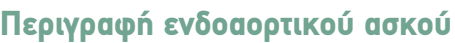

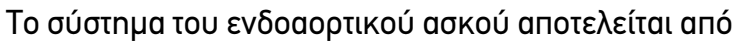

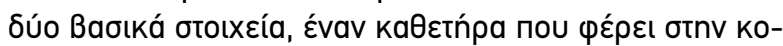

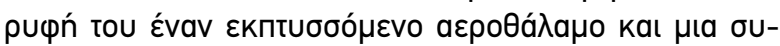

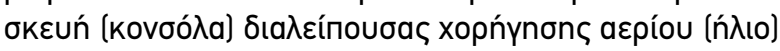

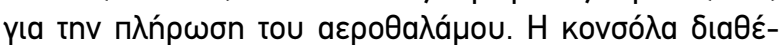

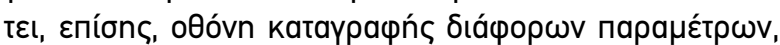

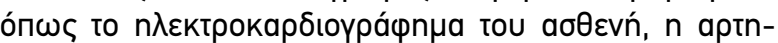

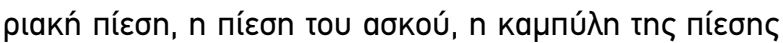




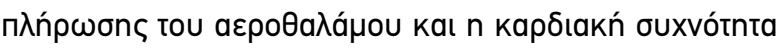

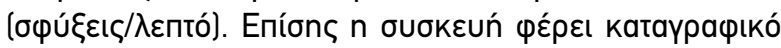

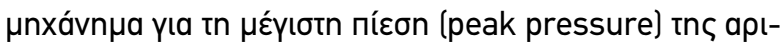

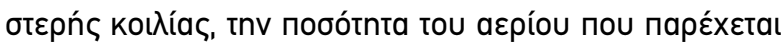

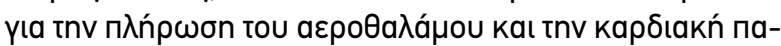
poxń.

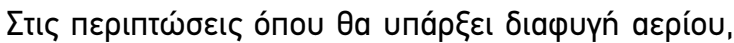

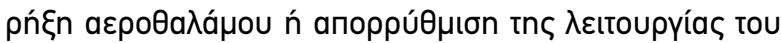

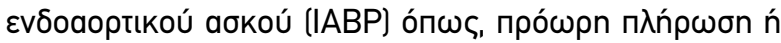

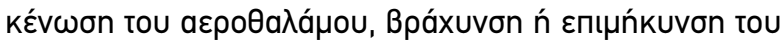

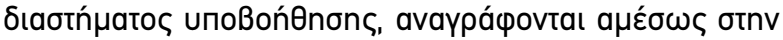

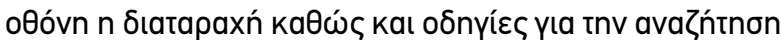
tnc attías tnc ठıatapaxńs autńs (EıKóva 1).

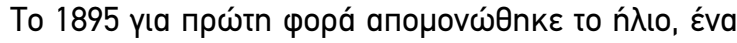

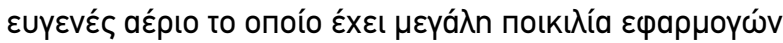

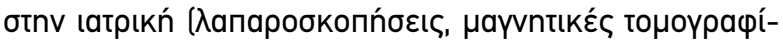

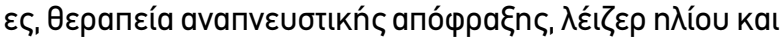

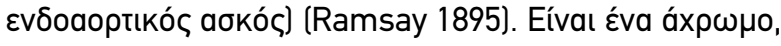

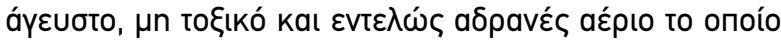

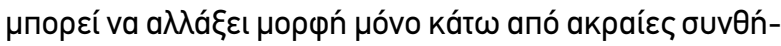

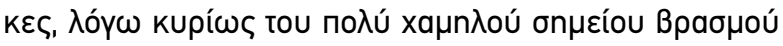

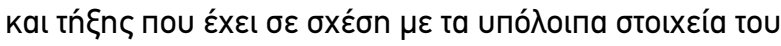

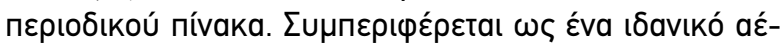

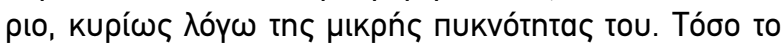

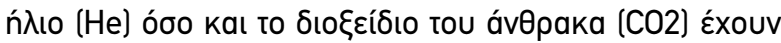

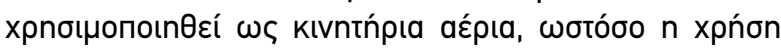

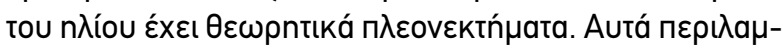

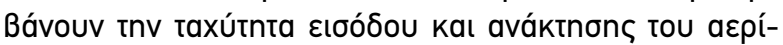

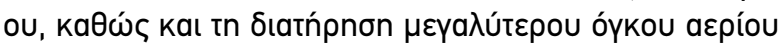

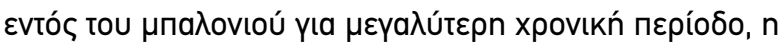

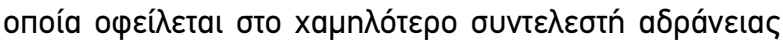

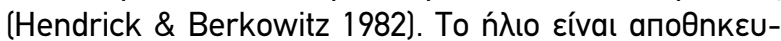

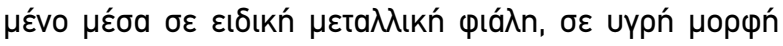

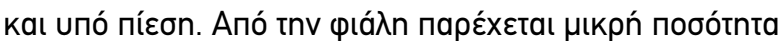

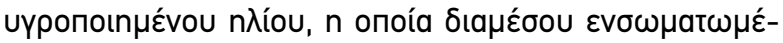

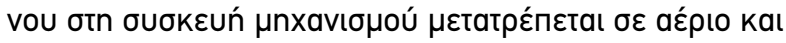

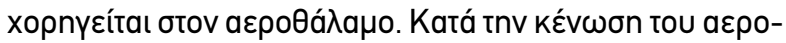

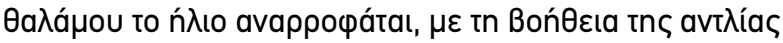

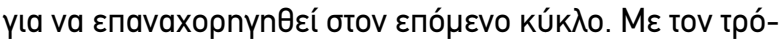

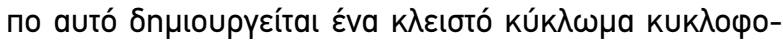

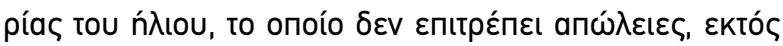

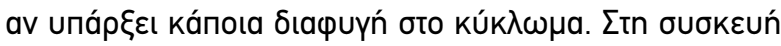

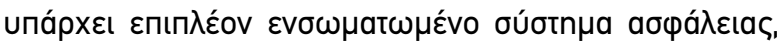

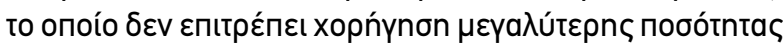

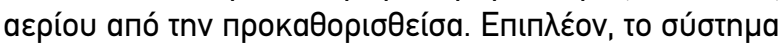

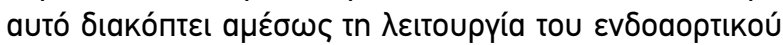

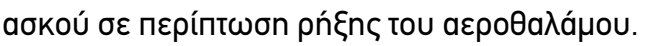

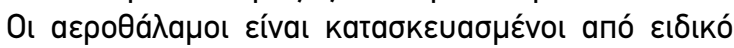

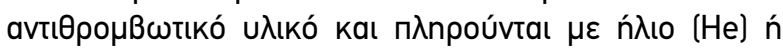

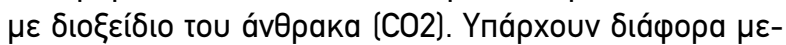

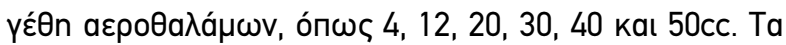

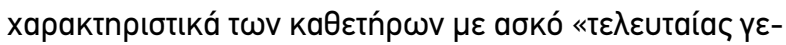

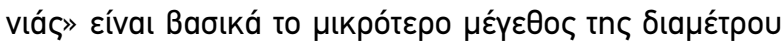

Eıkóva 1.

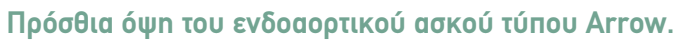

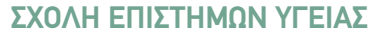
ikee.lib.auth.gr

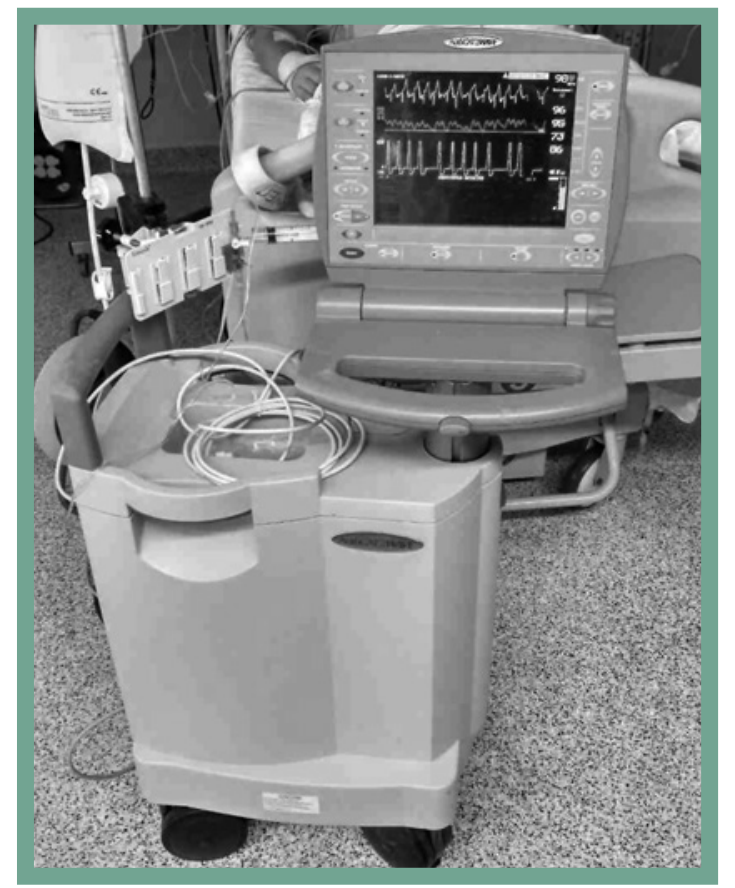

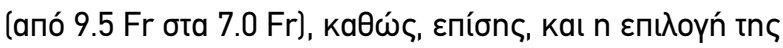

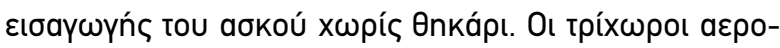

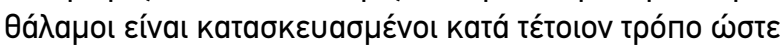

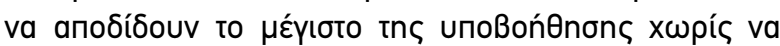

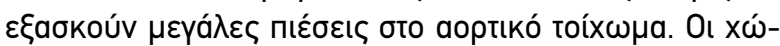

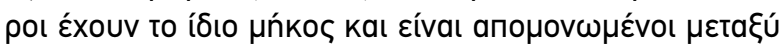

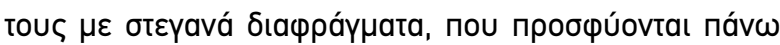

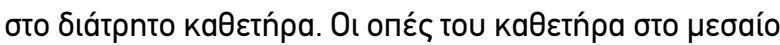

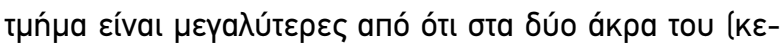

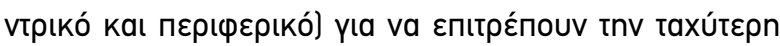

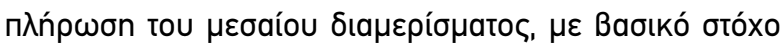

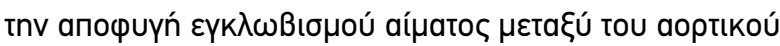

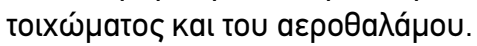

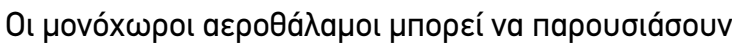

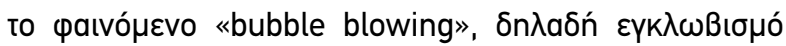

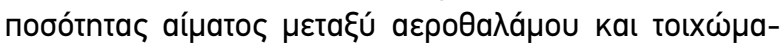

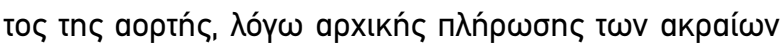

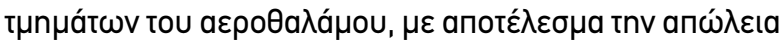

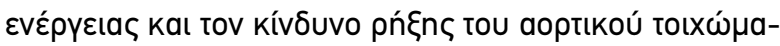

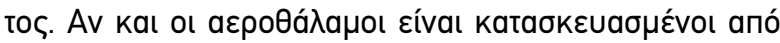

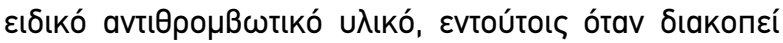

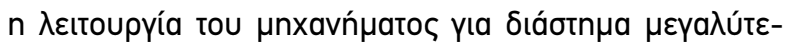

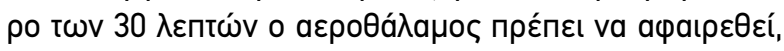

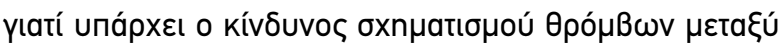

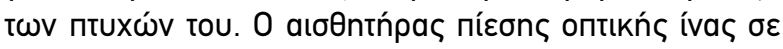

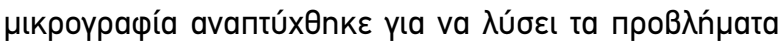




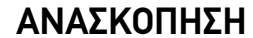

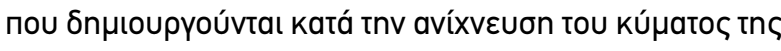

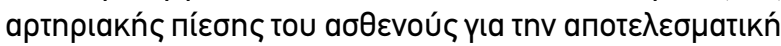

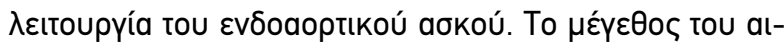

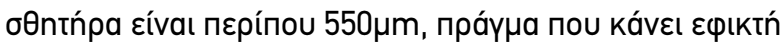

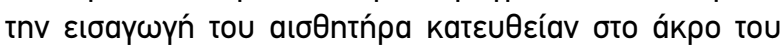

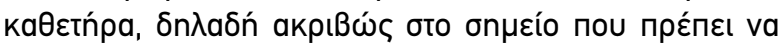

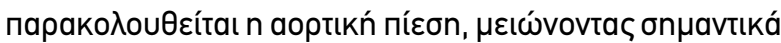

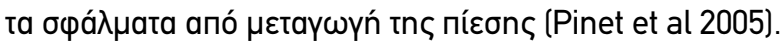

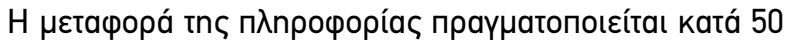

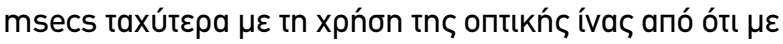

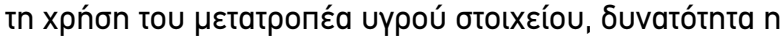

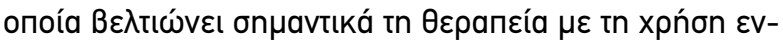

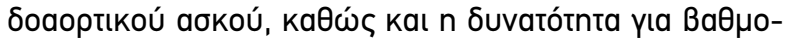

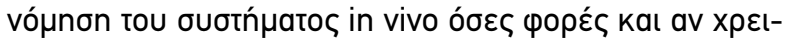

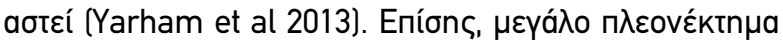

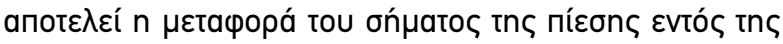

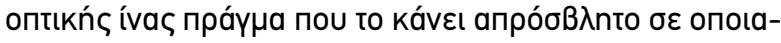

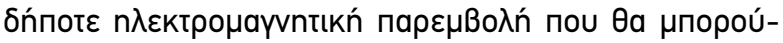

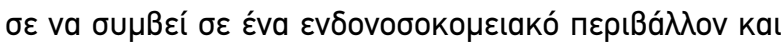

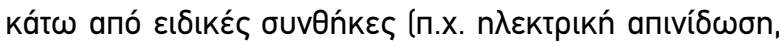

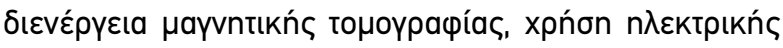

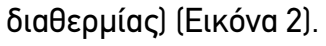

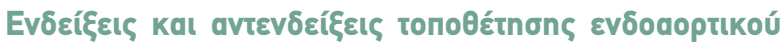 वซKoú}

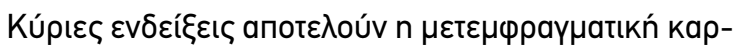

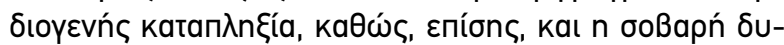

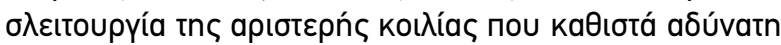

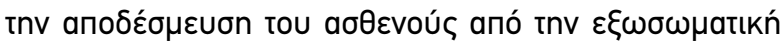

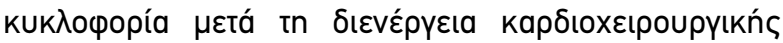

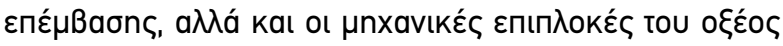

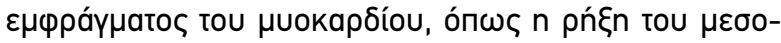

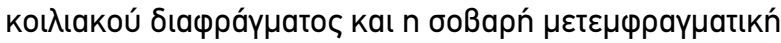

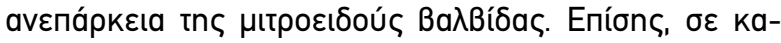

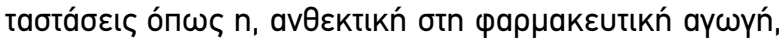

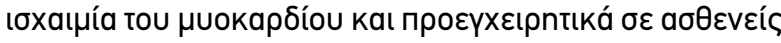

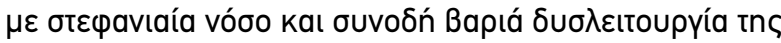

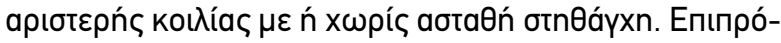

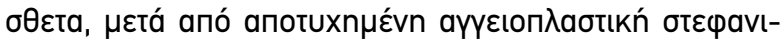

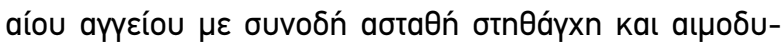

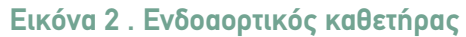

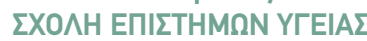 ikee.lib.auth.gr}

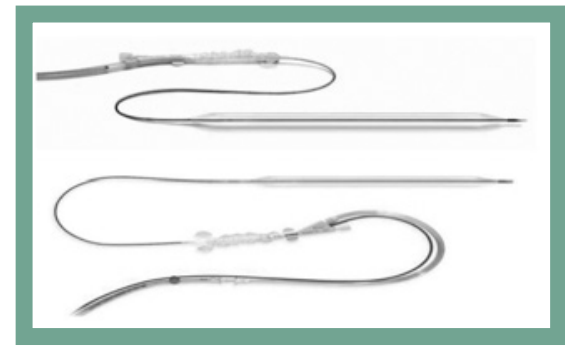

Eıкóva 3.

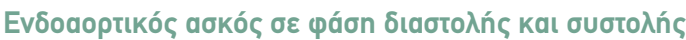
IABP (Intra-Aortic Balloon Pump ) nmcheartcare.ae

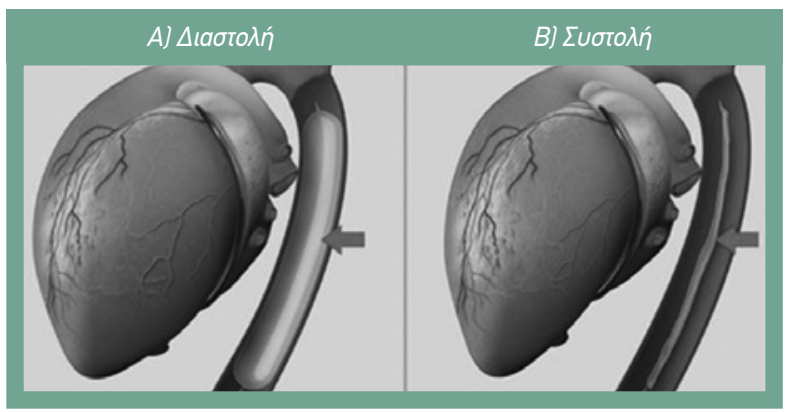

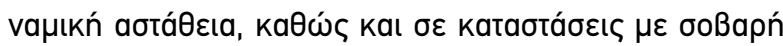

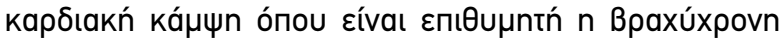

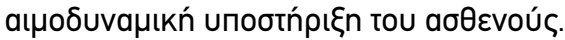

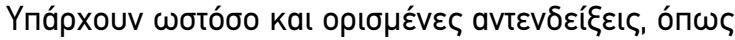

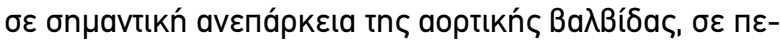

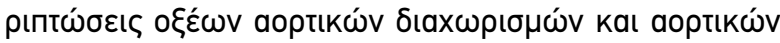

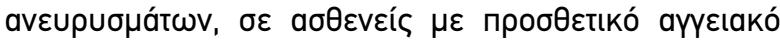

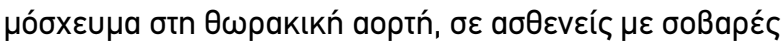

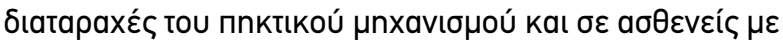

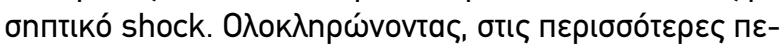

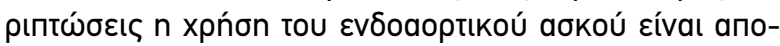

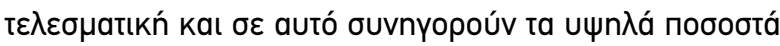

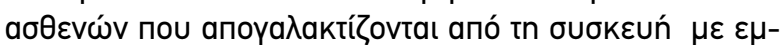

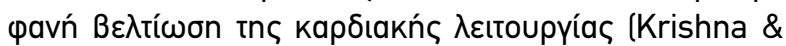
Zacharowski 2009).

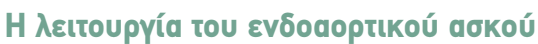

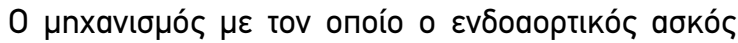

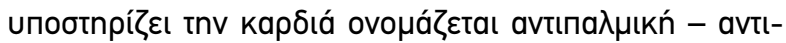

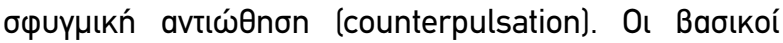

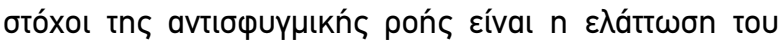

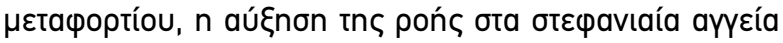
( n $\mu \varepsilon^{i} \omega \sigma n$ tn

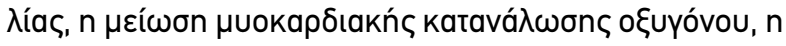

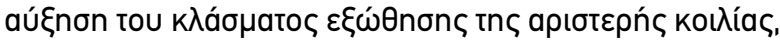

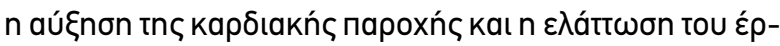

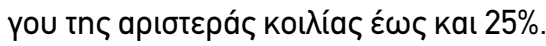

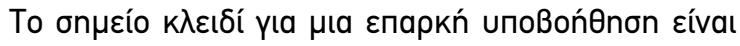

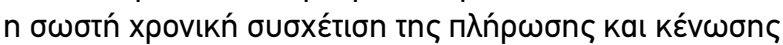

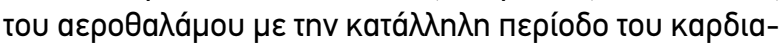

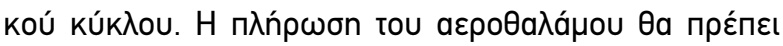

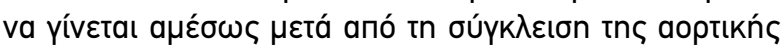

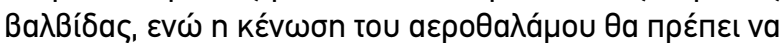

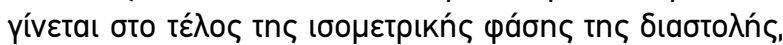

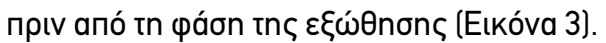

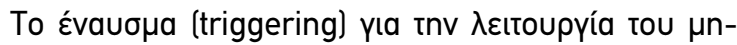

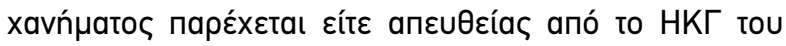


Etkóva 4. Xpoviouós tou ađkoú

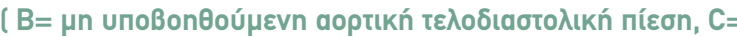

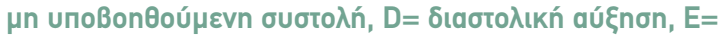

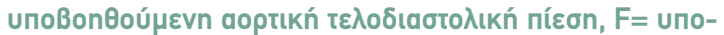

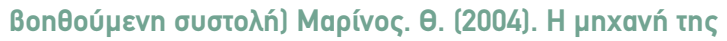

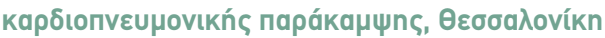

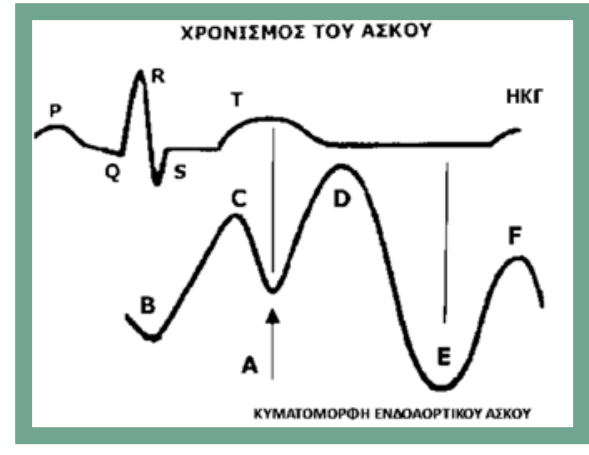

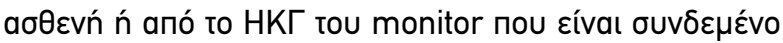

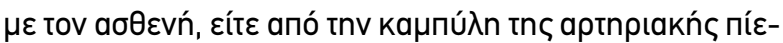

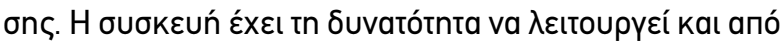

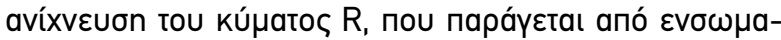

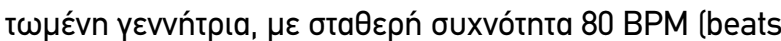
per minutes).

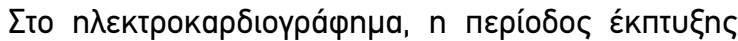

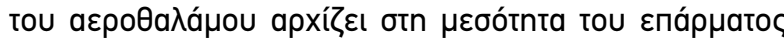

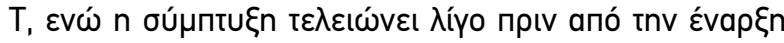

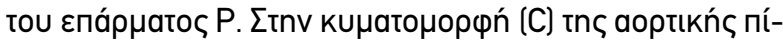

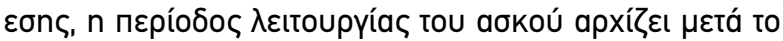

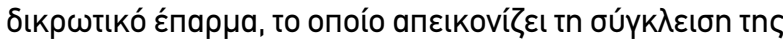

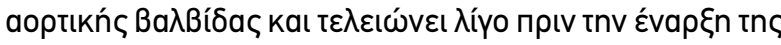

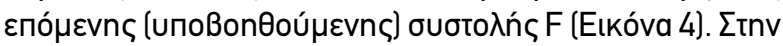

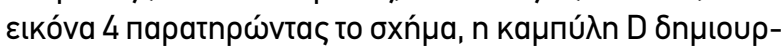

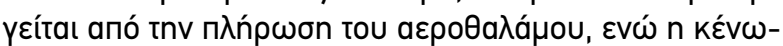

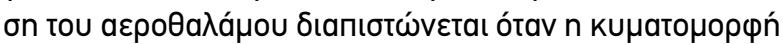

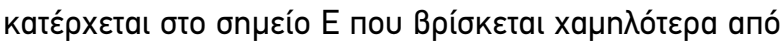

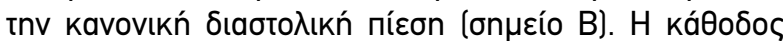

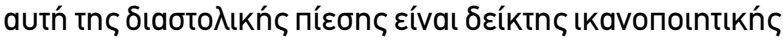

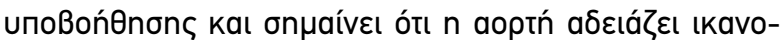

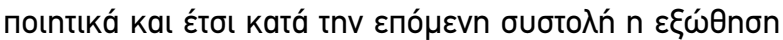

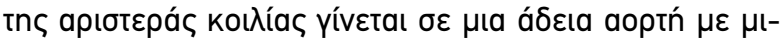

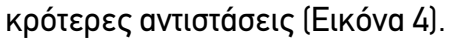

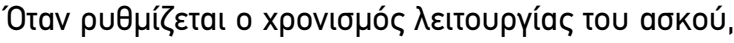

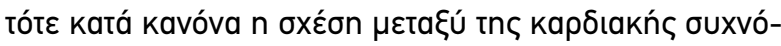

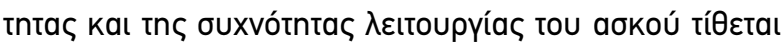

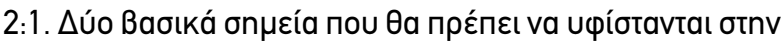

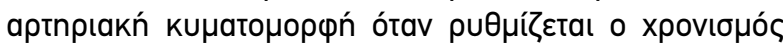

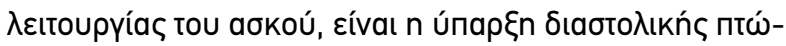

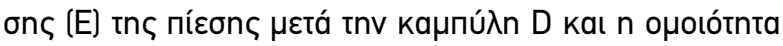
$\mu \varepsilon t a \xi u ́$ tn

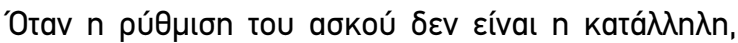
tót

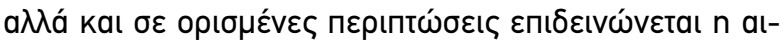

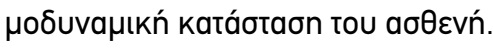

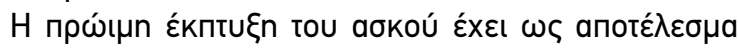

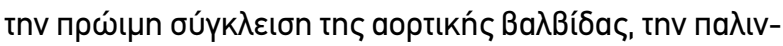

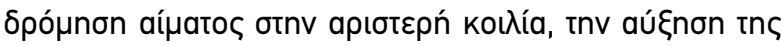

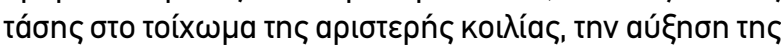

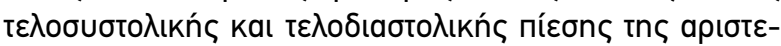

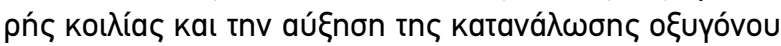

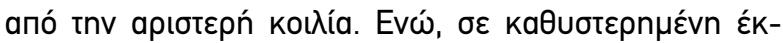

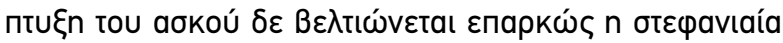

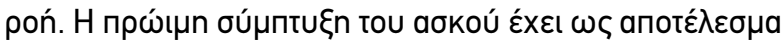

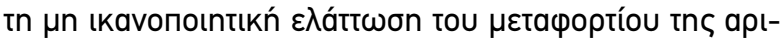

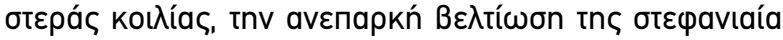

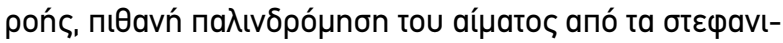

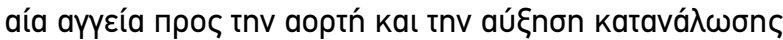

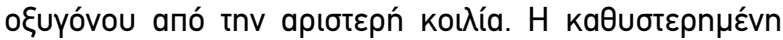

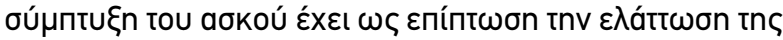

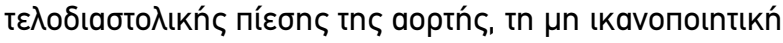

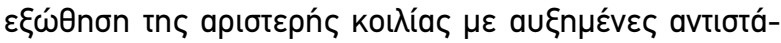

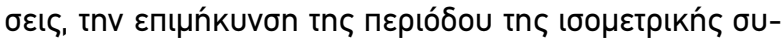

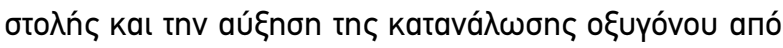

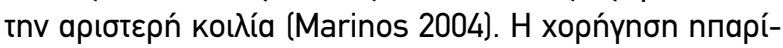

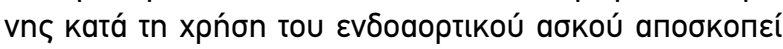

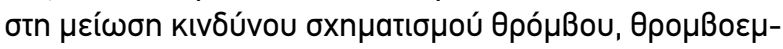

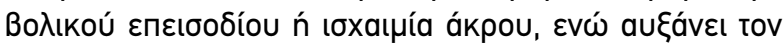

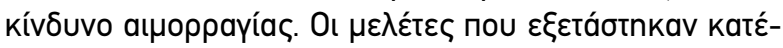

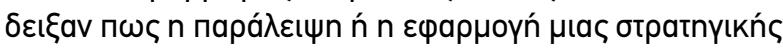
$\varepsilon \Pi \iota \lambda \varepsilon k t$ เkń

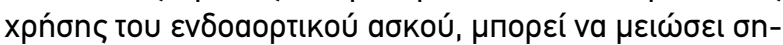

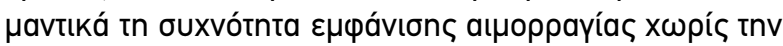

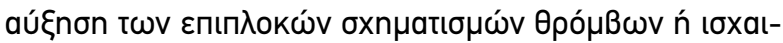

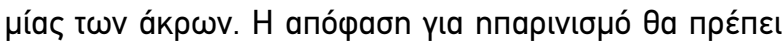

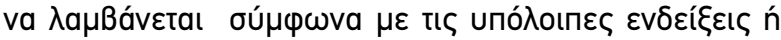

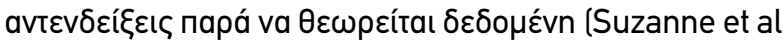
1997, Pucher et al 2012).

\section{Епเп入оке́}

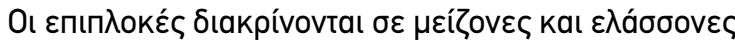

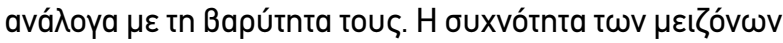

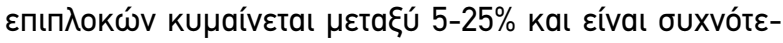

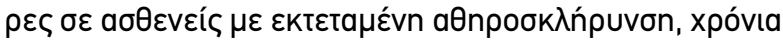

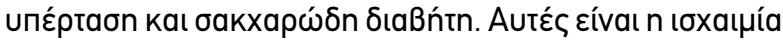

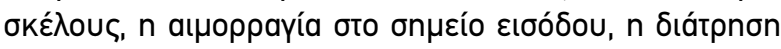

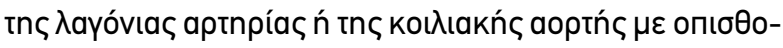

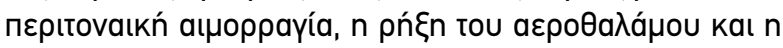

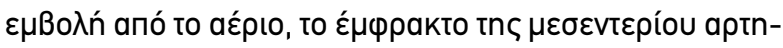

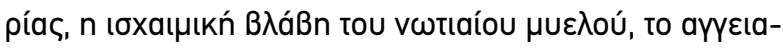

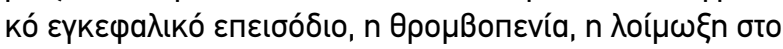

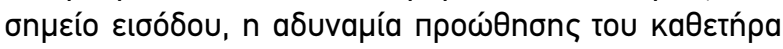

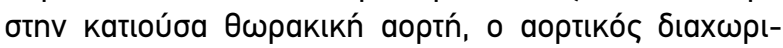

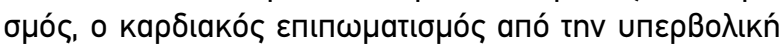

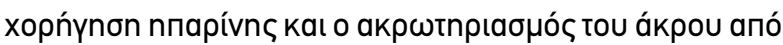

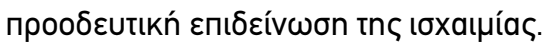

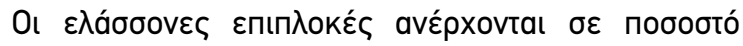




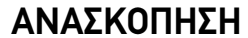

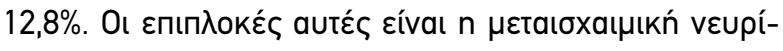

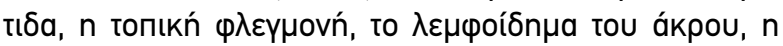

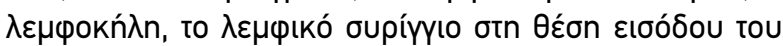

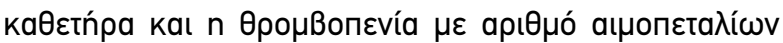

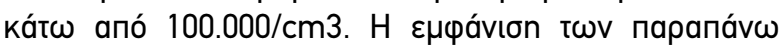

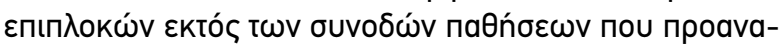

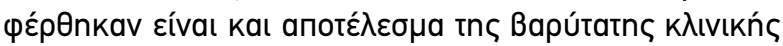

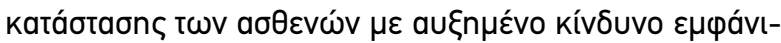

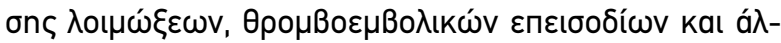

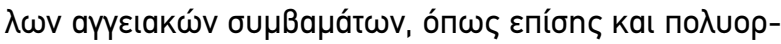

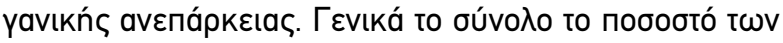

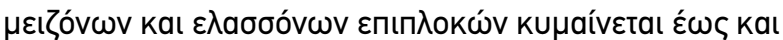

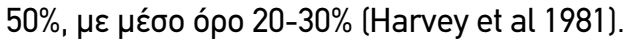

\section{EPEYNHTIKA $\triangle E \triangle O M E N A$}

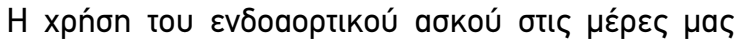

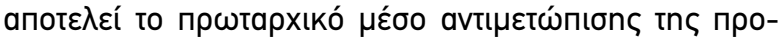

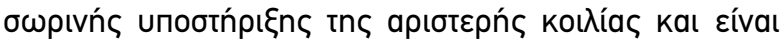

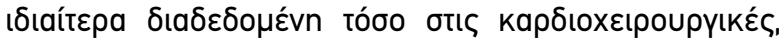

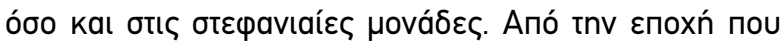

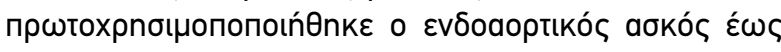

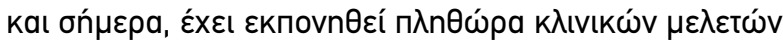

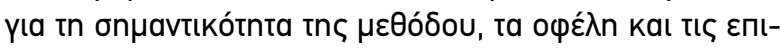

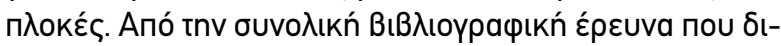

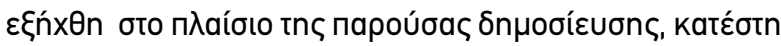

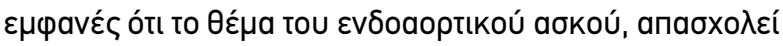

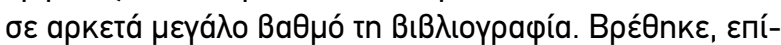

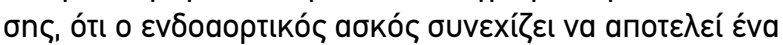

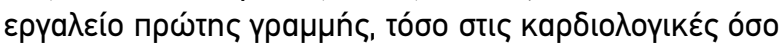

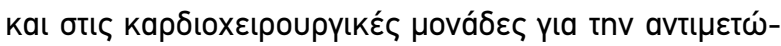

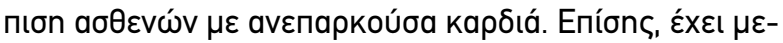

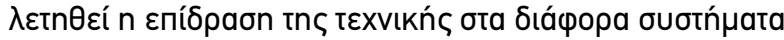

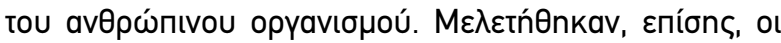

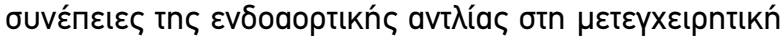

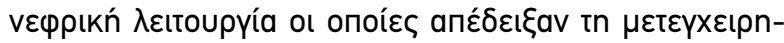

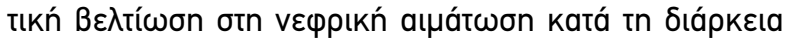
avtiẃӨnons (Hilberman et al 1981). Eníons, to 1992, $\mu \varepsilon-$

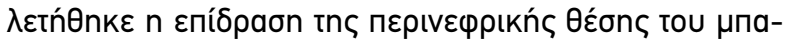

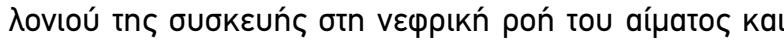

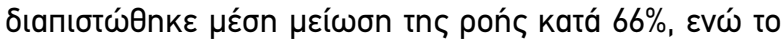

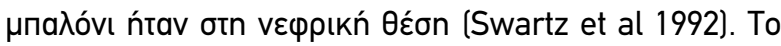

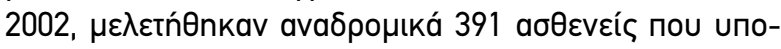

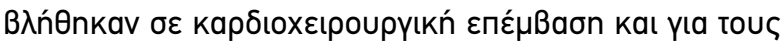

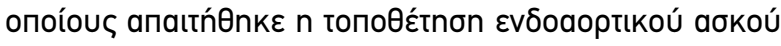

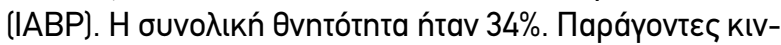

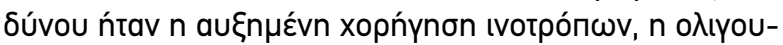

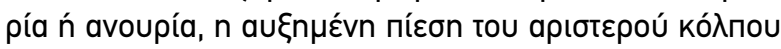

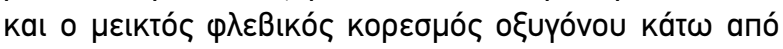
$60 \%$ (Hausmann et al 2002).

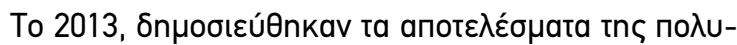

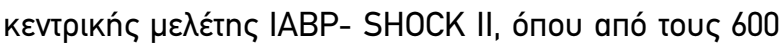

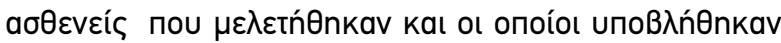

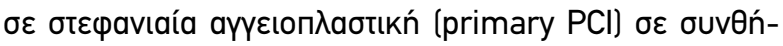

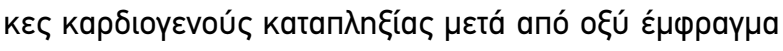

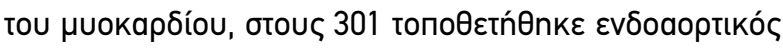

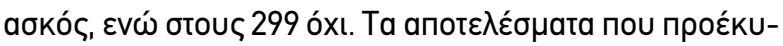

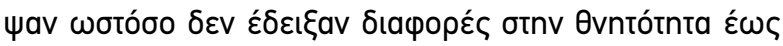

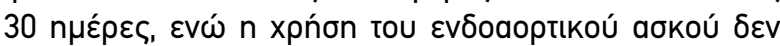

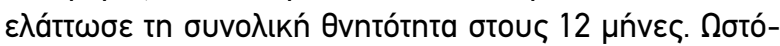

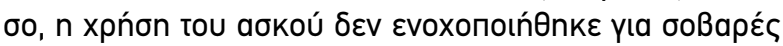

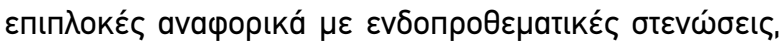

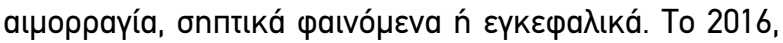

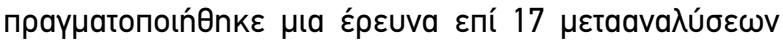

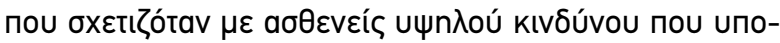

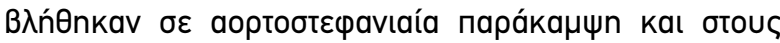

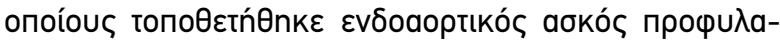

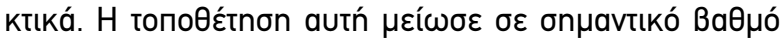

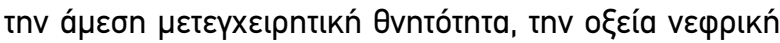

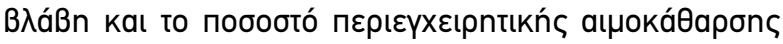

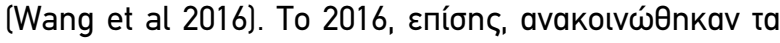

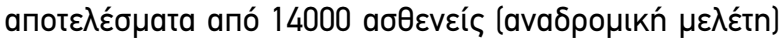

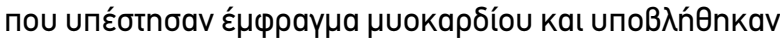

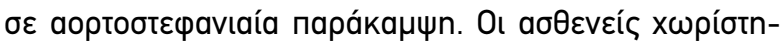

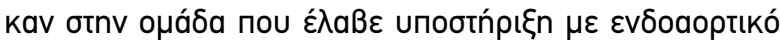

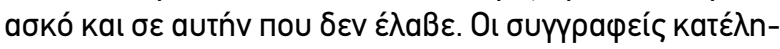

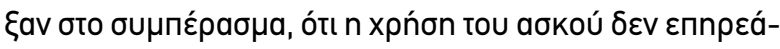

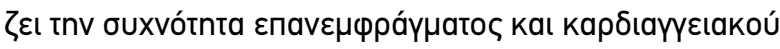

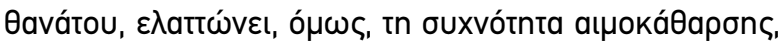

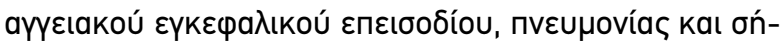

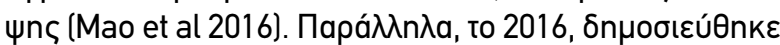

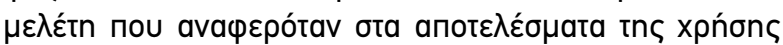

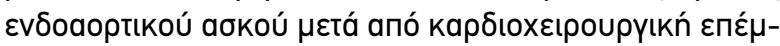

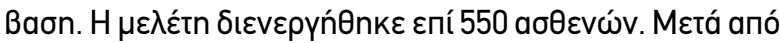

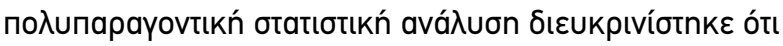

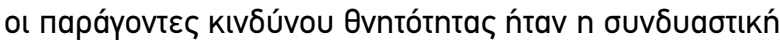

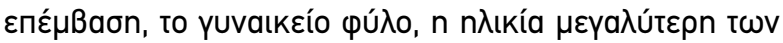

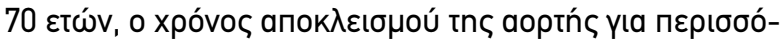

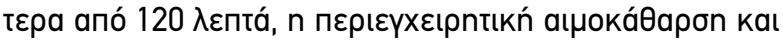

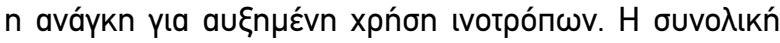

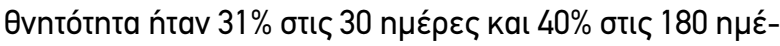

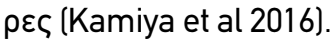

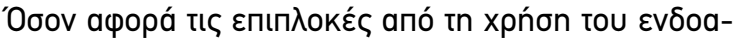

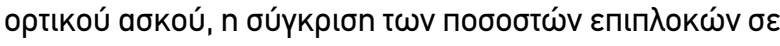

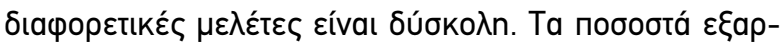

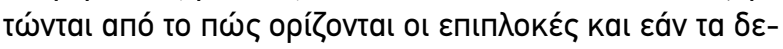

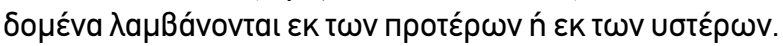

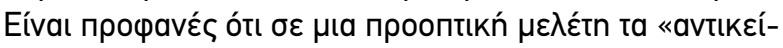

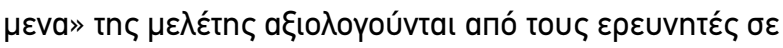

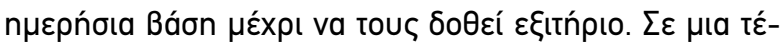

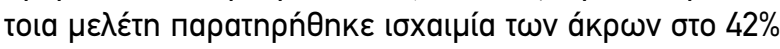

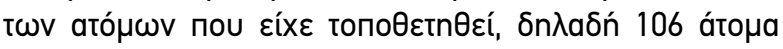

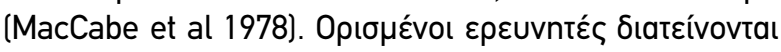

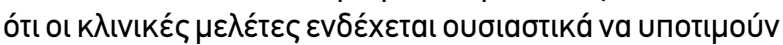

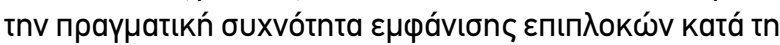

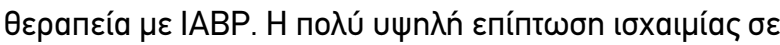

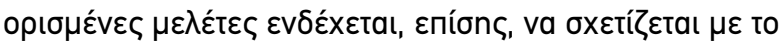




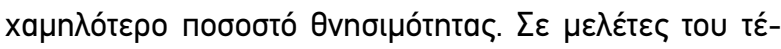

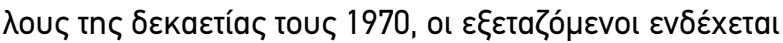

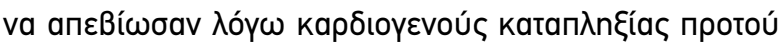

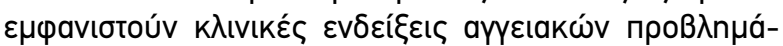

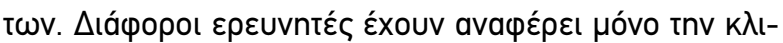

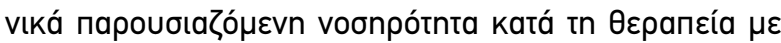

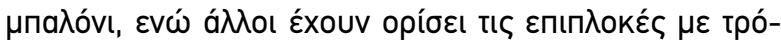

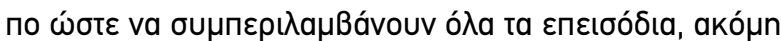

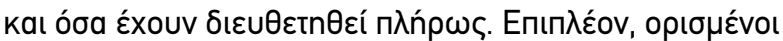
$\varepsilon \rho \varepsilon u v n t \varepsilon ́ \varsigma$ прох

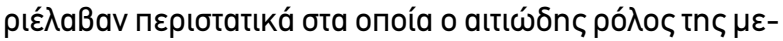

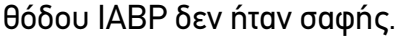

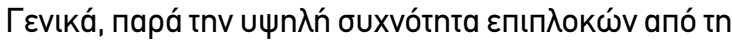

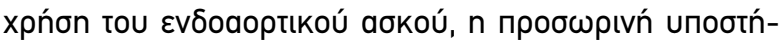

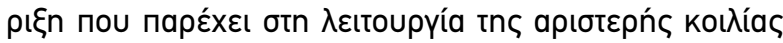

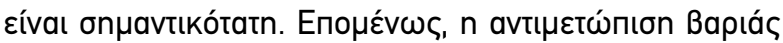

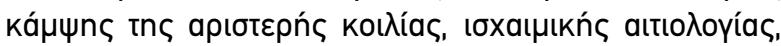

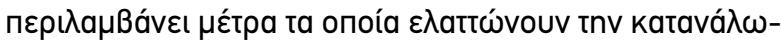

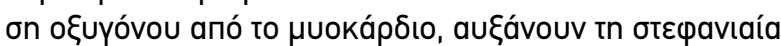

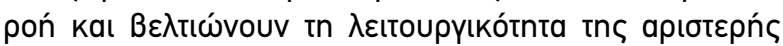

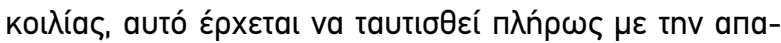

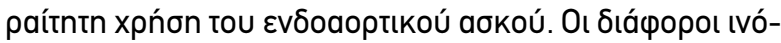

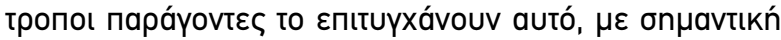

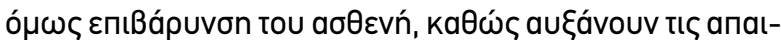

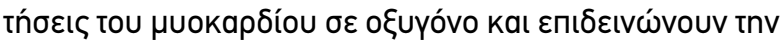

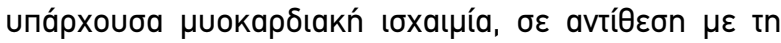

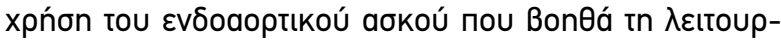

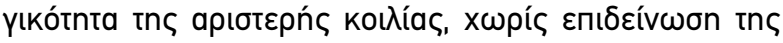
unápxovoas ıxxaıpíac.

\section{¿YMПEPAГMATA}

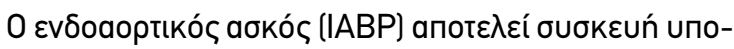

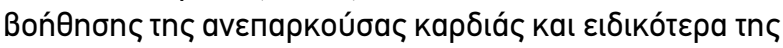

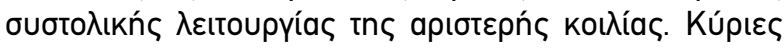

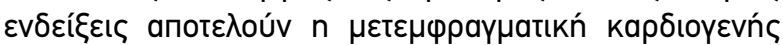

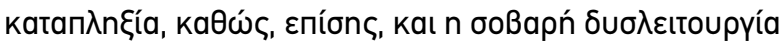

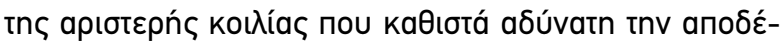

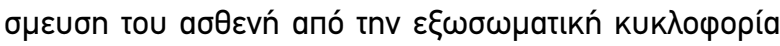

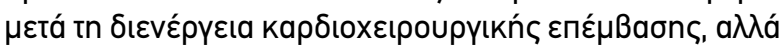

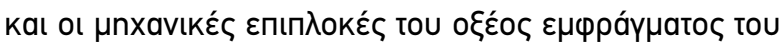

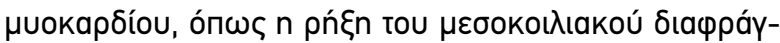

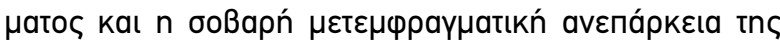

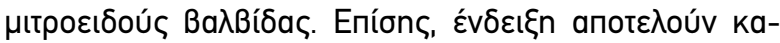

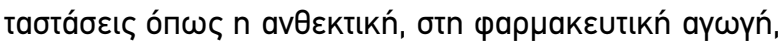

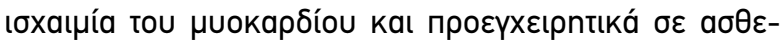

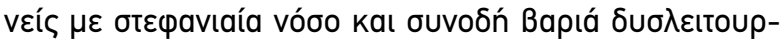

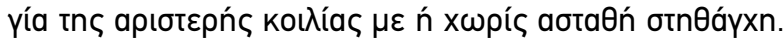

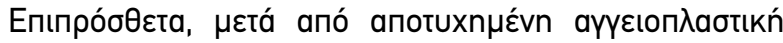

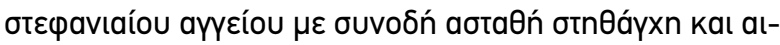

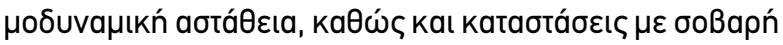

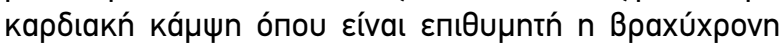

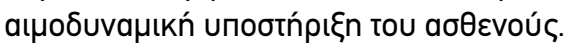

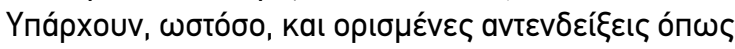

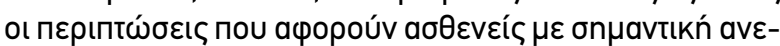

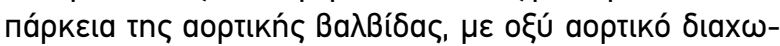

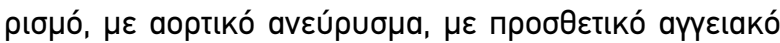

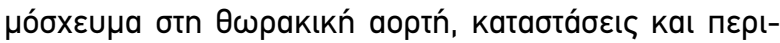

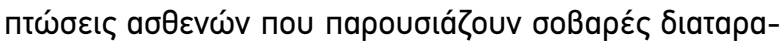

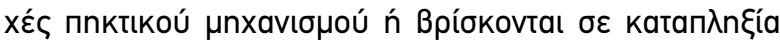
(shock).

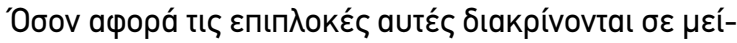

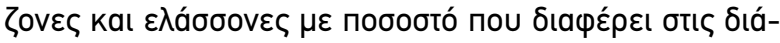

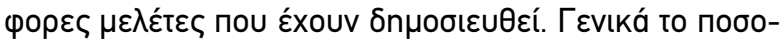

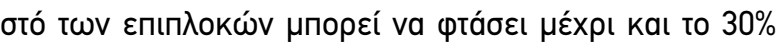
tou ouvóגou.

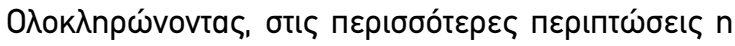

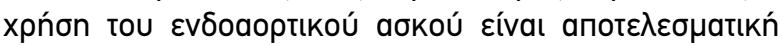

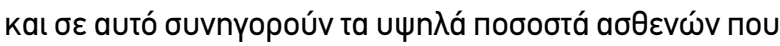

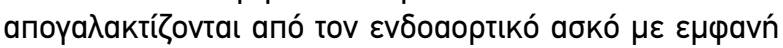

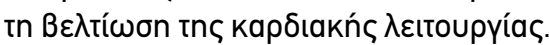




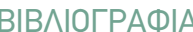

Bregman D., Kripke D.C. \& Goetz R.H. (1970). The effect of synchronous unidirectional intra-aortic ballon pumping of hemodynamics and coronary blood flow in cardiogenic shock. Transactions American Society for Artificial Internal Organs 16:439.

Buckley M.J., Leinbach R.C., Kastor J.A., Laird J., Kantrowitz A., Madras P., Sanders C. \& Austen G. (1970). Hemodynamic evaluation of intra-aortic ballon pumping in man. Circulation 46:130.

Clauss R.H., Missler P., Reed G.E. \& Tice D. (1962). Assisted circulation by counterpulsation with an Intraorticballon. Methods and effects. In digest, 15th Annual conference on engineering in Medicine and Biology. American Institute of Electrical Engineers Instruments Society of America 4:44.

Ficek S.J., Stammers A., Deligonul U., Shurmur S.W., Alonso A. \& Glbraith T. (1997). Hemastatic assessment of patients undergoing intra-aortic balloon pump therapy. Journal of Extracorporeal Technology 9(2): 78-82.

Harken D.E. (1958). Presentation at the International College of Cardiology Meeting. Brussels, Belgium 2:14-23.

Harvey J.C., Goldstein J.T., McCabe J.C., Hoover E.L., Gay W.A. Jr \& Subramanian V.A. (1981). Complications of percutaneous intraaortic balloon pumping, Circulation 2:114.

Hausmann H., Potapov E.V., Koster A., Krabatsch T., Stein J., Yeter R., Kukucka M., Sodian R., Kuppe H. \& Hetzer R. (2002). Prognosis after the implantation of an intra-aortic balloon pump in cardiac surgery calculated with a new score. Circulation 106:203-6.

Hendrick H.L. \& Berkowitz D. (1982). The differences between intraaortic balloon pumps and their use. Critical Care Medicine 10:796

Hilberman M. (1981). Effect of the intra-aortic balloon pump upon postoperative renal function in man, Critical Care Medicine 9:85.

Kamiya H., Schilling M., Akhyari P., Ruhparwar A., Kallenbach K., Karck M. \& Lichtenberg A. (2016). Outcome analysis for prediction of early and long-term survival in patients receiving intra-aortic balloon pumping after cardiac surgery. General thoracic and cardiovascular surgery, 64(10): 584-591.

Kantrowitz A., Akutsu T., Chaptal P.A., Krakauer J., Kantrowitz A.R. \& Jones R.T. (1966). A clinical experience with an implanted mechanical auxiliary ventricle. JAMA, 197(7): 525-529.

Kantrowitz A. \& McKinnon W.P. (1958). The experimental use of the diaphragm as an auxiliary myocardium. Sug Forum 9:266.

Katrowitz A., Krakauer J.S., Rosebaum A., Butner A.N., Freed P.S \& Jaron D. (1969). Phase- shift ballon pumping in medically refractory cardiogenic shock: results in 27 patients. Arch Surgery 99: 739.

Kantrowitz A., Tjonneland S., Freed P.S., Phillips S.J., Butner A.N. \& Sherman J.L. (1968). Initial clinical experience with intra-aortic ballon pumping in cardiology shock. JAMA 203:135.

Krishna M. \& Zacharowski, K. (2009). Principles of intra-aortic balloon pump counterpulsation. Continuing Education in Anaesthesia Critical Care \& Pain, 9(1): 24-28.

MacCabe J.C., Abel R.M., Subramanian V.A. \& Guy W.A (1978). Complications of intra-aortic balloon insertion and counterpulsation, Circulation 57:769

Marinos T. (2004). The heart and lung machine 14:125

Mao C.T., Wang J.L., Chen D.Y., Tsai M.L., Lin Y.S., Cherng W.J., Wand C.H., Wen M.S., Hsieh I.C., Hung M.J., Chen C.C. \& Chen T.H. (2016). Benefits of Intraaortic Balloon Support for Myocardial Infarction Patients in Severe Cardiogenic Shock Undergoing Coronary Revascularization. PLOS One 11:145-171.

Moulopoulos S.D., Topaz S. \& Kolff W.J. (1962). Diastolic ballon pumping (with carbon dioxide) in the aorta- A mechanical assistance to the failing circulation. American Heart Journal 63:669.

Moulopoulos S.D., Topaz S. \& Kolff W.J. (1962). Extracorporeal assistance to the circulation and intraortic ballon pumping. Transactions of the American Society for the Artificial Internal Organs 8: 85

Pinet É., Pham A. \& Rioux S. (2005). Miniature fiber optic pressure sensor for medical applications: an opportunity for intra-aortic balloon pumping (IABP) therapy. Proceedings of SPIE Vol 5855: 235.

Pucher P.H., Cummings G.I., Shipolini A.R., McCormack D.J. et al (2012). Is heparin needed for patients with an intra-aortic balloon pump? Interact Cardiovasc Thoracic Surgery 15(1): 136-139.

Ramsay W. (1895). Helium, a gaseous constituent of certain minerals. Part I. Proceedings of the Royal Society of London, 58(347-352), pp.80-89.

Stamatelopoulos S., Boufas D., Nanas I., Sideris D., Pistefthos A., Lolas X. \& Moulopoulos S. (1978). Clinical application of aortic pump in two cases of cardiogenic shock. Greek Cardiology Review 19: 209-2013

Swartz M.T., Sakamoto T., Arai H., Reedy J.E., Salenas T., Yuda T., Standeven J.W., \& Pennington G. (1992). Effects of intraaortic balloon position on renal artery blood flow, The Annals of Thoracic Surgery 53: 604

Wang J., Yu W., Gao M., Gu C. \& Yu Y. (2016). Preoperative Prophylactic Intraaortic Balloon Pump Reduces the Incidence of Postoperative Acute Kidney Injury and Short-Term Death of High-Risk Patients Undergoing Coronary Artery Bypass Grafting: A Meta-Analysis of 17 Studies. The Annals of Thoracic Surgery 101: 2007-19.

Yarham G., Clements A., Morris C., Cumberland T., Bryan M., Oliver M., Burrows H. \& Mulholland J. (2013). Fiber-optic intra-aortic balloon therapy and its role within cardiac surgery. Perfusion 28(2): 97-102 


\title{
The use of Intra-Aortic balloon pump as a left ventricle assist device
}

\author{
Evlabia Stroggili ${ }^{1}$, Stylianos Mimikos ${ }^{2}$, George Karapanagiotidis ${ }^{3}$ \\ 1. RN, MSc, Saint Luke Clinic, Thessaloniki \\ 2. RN, MSc University Hospital of Thessaloniki AHEPA \\ 3. MD, Assistant Professor of Cardiothoracic Surgery, Aristotle University of Thessaloniki
}

\begin{abstract}
The ischemic heart failure constitutes one of the commonest reasons of death nowadays, mainly in the western hemisphere. According to scientific predictions the number of deaths will be aggravated in the foreseeable and distant future. Its management is a separate field of cardiology and cardiothoracic surgery which includes medication, cardiothoracic surgeries and the insertion and use of devices that assist the heart function, culminating in the implantation of an artificial heart and transplantation. Among the mechanical means available for the support of the insufficient cardiac function, the first aid is provided by the Intra-Aortic Balloon Pump (IABP). The IABP constitutes a practical, efficient and low-cost device which is widely used in coronary and cardiothoracic care units all over the world. It provides significant facilitation of the left ventricle that is failing due to ischemia or myocardial infarction. It is inserted intravascularly and it has absolute indications and contraindications. There are various complications that can occur, however its contribution to the assistance of the left ventricle outweighs any problem that might be caused. The aim of the present paper is to present, with the help of historic and literature data, the basic principles of use and function of the IABP and its possible side effects.
\end{abstract}

Key Words: Intra-Aortic Balloon Pump, Ischemic Heart Failure, Ischaemia

Corresponding author: Stroggili Evlabia

e-mail: evlabia1@gmail.com
Submission date: $13 / 11 / 18$ Re-submission date: 01/10/2019 Publication date: April 2020

Citation: Stroggili E., Mimikos S. \& Karapanagiotidis G. (2020). The use of Intra-Aortic balloon pump as a left ventricle assist device. Hellenic Journal of Nursing Science 13(2): 5-13, https://doi.org/10.24283/hjns.202022 